\title{
DETERMINATION OF RECEIVER SUSCEPTIBILITY TO RADIO FREQUENCY INTERFERENCE FROM PORTABLE ELECTRONIC DEVICES
}

\author{
Truong X. Nguyen and Jay J. Ely, NASA Langley Research Center, Hampton, VA
}

\begin{abstract}
With the increasing pressures to allow wireless devices on aircraft, the susceptibility of aircraft receivers to interference from Portable Electronic Devices (PEDs) becomes an increasing concern. Many investigations were conducted in the past, with limited success, to quantify device emissions, path loss, and receiver interference susceptibility thresholds. This paper outlines the recent effort in determining the receiver susceptibility thresholds for ILS, VOR and GPS systems. The effort primarily consists of analysis of data available openly as reported in many RTCA and ICAO documents as well as manufacturers data on receiver sensitivity. Shortcomings with the susceptibility threshold data reported in the RTCA documents are presented, and an approach for an indepth study is suggested.

In addition, intermodulation products were observed and demonstrated in a laboratory experiment when multiple PEDs were in the proximity of each other. These intermodulation effects generate spurious frequencies that may fall within aircraft communication or navigation bands causing undesirable effects. Results from a preliminary analysis are presented that show possible harmful combinations of PEDs and the potentially affected aircraft bands.
\end{abstract}

\section{Introduction}

With the growing use of portable electronic devices (PEDs) on board aircraft, the potential interference with sensitive aircraft receiver systems is an increasing concern. Past efforts reported in DO-199 [1] and DO-233 [2] assess the potential of interference to aircraft receivers from nonintentionally transmitting PEDs such as laptop

This work was funded in part by the FAA Aircraft Certification Office and by NASA's Aviation Safety Program Single Aircraft Accident Prevention Project. computers and $\mathrm{CD}$ players. Those devices can easily be carried onboard an aircraft by passengers. The approach taken was to assess each of the elements, including source emissions, signal coupling pathloss, and victim's susceptibility threshold. The source emissions and the signal pathloss should produce signal below the susceptibility threshold of the victim system to avoid interference.

A more recent effort described in a companion paper [3] was an attempt to assess the threat to aircraft receivers from cellular phones in particular. The work will expand in the future to include other wireless devices such as IEEE 802.11a, b wireless LANs, Bluetooth devices and FRS/GMRS radios. These intentional transmitting devices tend to have much higher FCC out-off-band emission limits, typically $-43 \mathrm{~dB}$ from peak in-band emissions, than the limits described in FCC Part 15 for nonintentional transmitters. Therefore, these devices may pose higher risks if undesirable signals coupled into the antenna ports of aircraft receiver systems.

Following the same approach used in [1] and [2], the work in [3] measured source RF emission from eight cellular phones of three different technologies - CDMA, GSM and AMPS - in the Localizer, Glideslope, VOR and GPS bands. In addition, [3] also includes a summary of pathloss data recently collected on several different aircraft for many communication and navigations bands. The extensive new set of pathloss data [4], along with others currently being collected and analyzed, were made possible through the cooperative agreements with major airlines who allowed access to their aircraft along with providing supporting staff.

The effort presented in this paper is a part of work described in [3] in an attempt to get a better understanding on susceptibilities receivers, or the victim systems. For Localizer, Glideslope and VOR, the paper first describes the desired signal

\section{U.S. Government work not protected by U.S. Copyright}


strength in the coverage airspace. The desired signal strengths at the receivers are then shown based on the computed values reported in various standards and receiver Minimum Operational Performance Standards (MOPS). The desired signal strength data is then compared with the results from a survey of receiver sensitivities. These receiver sensitivities are related to receiver susceptibility thresholds for certain types of modulated interference signals according to DO-233. Receiver susceptibility data are then summarized from DO-199 and DO-233. Deficiencies in the currently available data are pointed out for future efforts.

For GPS, receiver susceptibilities are well defined as reported in various ITU and receiver MOPS. This paper summarizes the elements relevant to PED problems.

In addition, intermodulation products (IM) that fall in aircraft receiver bands, caused by the presence of multiple cellular phones, are demonstrated in a laboratory measurement. Further analysis also shows the potential interference from intermodulation products due to various other combinations of wireless devices.

\section{Localizer, Glideslope and VOR Susceptibility Thresholds}

In this section, the minimum field environments assumed in various specifications are summarized and compared, and the desired signal strengths at the receivers are reported. The resulting desired signal strengths are then compared with the results from a survey of receiver sensitivities provided by receiver manufacturers. The susceptibility thresholds, in relation to desired signal strength at the receiver, are summarized from the previous works documented in RTCA/DO-199 and DO-233. The results together with limitations on their usefulness are discussed.

\section{Localizer, Glideslope and VOR Minimum Field Environment}

The minimum field environments within the airspace coverage volume are used in estimating the desired signal field strength at the receiver. The susceptibility thresholds can then be calculated if the desired to undesired signal ratio is known.
The minimum field environments were derived from many documents, including the ICAO Annex 10 [5], receiver Minimum Operational Performance Standards (MOPS) RTCA/DO 192, 195, 196 [6,7,8], and RTCA/DO-199 [1], RTCA/DO-233 [2] for aircraft interference by PEDs. The results are tabulated in Table 1.

Table 1. Minimum Field Environment Within Coverage Airspace

\begin{tabular}{|l|c|c|c|}
\hline & $\begin{array}{c}\text { ILS-Loc } \\
(\mathbf{u V} / \mathrm{m})\end{array}$ & $\begin{array}{c}\text { IL-GS } \\
(\mathrm{uV} / \mathrm{m})\end{array}$ & $\begin{array}{c}\text { VOR } \\
(\mathrm{uV} / \mathrm{m})\end{array}$ \\
\hline ICAO & 40 & 400 & 90 \\
\hline $\begin{array}{l}\text { RTCA/DO- } \\
\text { 192,195,196 }\end{array}$ & 40 & 350 & 20 \\
\hline RTCA/DO-199 & 40 & 400 & 20 \\
\hline RTCA/DO-233 & 40 & 200 & 90 \\
\hline
\end{tabular}

As can be observed from Table 1, the minimum Localizer environment is the same in all listed documents. However, for Glideslope and VOR, the environments are different depending on the documents used.

Of all the sources listed, the field environment data documented in DO-192, 195 and 196 was most credible for the U.S. airspace as these documents are specified in FAA's Technical Standards Orders (TSOs) C36, C34 and C40 for Localizer, Glideslope and VOR instruments respectively.

\section{Desired Signals at Receivers}

Minimum desired signal strengths at the receiver inputs are documented in various documents such as receiver DO-192, 195, 196 and in DO-199, DO-233 for Portable Electronic Devices Carried on Board Aircraft. The results are summarized in Table 2.

As can be observed from Table 2, the calculated minimum signal strength at the receivers specified in DO-192, 195, 196, 199 and 233 vary from -86 to $-90 \mathrm{dBm}$ for Localizer, from -76 to $86 \mathrm{dBm}$ for Glideslope and between -90 to -97 $\mathrm{dBm}$ for VOR.

DO-192, 195, and 196 provided the data shown in Table 2 without clarifications. It is understood that they were computed assuming field incident upon an isotropic, lossless antenna, and 
that there was no additional cable loss. The same assumptions were made in DO-199 as the initial estimations, and the results were similar.

Table 2. Calculated Minimum Desired Signal Strength At Receiver Antenna Input And Comparison With Receiver Sensitivity

\begin{tabular}{|l|c|c|c|}
\hline & $\begin{array}{c}\text { ILS-Loc } \\
\text { (dBm) }\end{array}$ & $\begin{array}{c}\text { ILS-GS } \\
\text { (dBm) }\end{array}$ & $\begin{array}{c}\text { VOR } \\
\text { (dBm) }\end{array}$ \\
\hline $\begin{array}{l}\text { RTCA/DO- } \\
\text { 192,195,196 }\end{array}$ & -86 & -76 & -93 \\
\hline RTCA/DO-199 & -86 to -88 & -76 to -78 & -92 to -97 \\
\hline RTCA/DO-233 & -90 & -86 & -90 \\
\hline $\begin{array}{l}\text { Min/Max } \\
\text { Receiver } \\
\text { Sensitivity }\end{array}$ & $-113 /-93$ & $-99 /-87$ & $-113 /-99$ \\
\hline
\end{tabular}

* From Table 4

DO-199 also provided a range for minimum signal strength at receivers for each receiver system. For Localizer, the range is from $-86 \mathrm{dBm}$ at the output of a lossless isotropic antenna to $-88 \mathrm{dBm}$ to account for $2 \mathrm{~dB}$ cable loss. For Glideslope, the range is from $-76 \mathrm{dBm}$ at output of isotropic lossless antenna to $-78 \mathrm{dBm}$ for the additional $2 \mathrm{~dB}$ cable loss. For VOR, the range is from -92 at the output of the antenna to $-97 \mathrm{dBm}$, which accounts for the additional $2 \mathrm{~dB}$ cable loss and $3 \mathrm{~dB}$ splitter loss.

DO-233 on the other hand, estimated signal strength based on a number of corrections. These corrections included shadow loss, dipole antenna factor and aircraft cable loss. For shadow loss, DO233 assumed $3 \mathrm{~dB}$ for antennas at the bottom of an aircraft and $10 \mathrm{~dB}$ for antennas atop the aircraft. Aircraft antennas were assumed to behave like a monopole on a ground plane, thus having a dipole antenna factor. The cable loss was assumed to be 3 $\mathrm{dB}$. A sample calculation used in DO-233 is tabulated in Table 3.

For comparison, the last row of Table 2 also shows the range of receiver sensitivities according to manufacturers' published equipment specifications. These specifications were compiled from manufacturers' web sites and by communication with the manufacturers. More details are described in the next section.
Table 3. DO-233 Sample Desired Minimum Signal Strength Calculation

\begin{tabular}{|l|c|c|c|}
\hline & $\begin{array}{c}\text { ILS- } \\
\text { Localizer }\end{array}$ & $\begin{array}{c}\text { ILS- } \\
\text { Glide- } \\
\text { slope }\end{array}$ & VOR \\
\hline $\begin{array}{l}\text { External Signal } \\
\text { Strength } \\
\text { (dB } \mu \text { V/m) }\end{array}$ & 32 & 46 & 39 \\
\hline Shadow Loss (dB) & 3 & 3 & 10 \\
\hline $\begin{array}{l}\text { Dipole Antenna } \\
\text { Factor (dB) }\end{array}$ & 9 & 19 & 9 \\
\hline $\begin{array}{l}\text { Aircraft Cable } \\
\text { Loss (dB) }\end{array}$ & 3 & 3 & 3 \\
\hline $\begin{array}{l}\text { Desired Signal at } \\
\text { Receiver (dB } \mu \text { V/m) }\end{array}$ & 17 & 21 & 17 \\
\hline $\begin{array}{l}\text { Desired Signal at } \\
\text { Receiver (dBm) }\end{array}$ & $\mathbf{- 9 0}$ & $\mathbf{- 8 6}$ & $\mathbf{- 9 0}$ \\
\hline
\end{tabular}

The range of receiver sensitivity is from -113 to $-93 \mathrm{dBm}$ for Localizer, -99 to $-87 \mathrm{dBm}$ for Glideslope, and -113 to $-99 \mathrm{dBm}$ for VOR. Compared to the calculated minimum desired signal strengths, the receiver sensitivities are lower (more sensitive) in all cases, regardless whether the desired signal strengths were from receiver MOPS (such as DO-195, 196, 192), or from DO-199 and DO-233. This is very assuring that most receivers are sensitive enough in most installations.

\section{Manufacturer's Receiver Sensitivity Specifications}

Based on test results in DO-199 and DO-233, the receiver susceptibility thresholds bear a relationship with receiver sensitivity for certain types of modulated interference signal. Thus, it is desirable to characterize the receiver sensitivity as this may provide additional insights about the range of receiver interference thresholds.

A survey of receiver sensitivity was conducted for many commercially available models from large manufacturers of aircraft receivers, including Bendix King, Allied Signal, Honeywell, and Rockwell Collins. A summary of the results is shown in Table 4. The highest and the lowest values for each receiver are highlighted and underlined in the table.

As stated previously, there were wide ranges of sensitivity for each of the receivers. The difference in sensitivity between the most and the 
least sensitive models is $20 \mathrm{~dB}$ for Localizer, $12 \mathrm{~dB}$ for Glideslope and $14 \mathrm{~dB}$ for VOR. The large range of sensitivity, however, may be cause for concerns. It is possible that many receivers are too sensitive, which may lead to undesired valid signals being received far outside of the intended coverage airspace. The lower sensitivity threshold tends to imply lower interference thresholds, as the desired to undesired signal ratio tends to stay fixed according to DO-199 and DO-233. As a result, lower sensitivity threshold may lead to higher occurrences of false interference outside the intended coverage airspace.

Table 4. Receiver Sensitivity Derived From Equipment Specifications.

\begin{tabular}{|c|c|c|c|}
\hline \multicolumn{2}{|c|}{ GlideSlope } & \multicolumn{2}{|c|}{ Localteor } \\
\hline Model & Lavel (dBm) & Model & Level (dBm) \\
\hline \multicolumn{2}{|c|}{ 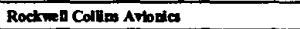 } & \multicolumn{2}{|c|}{ Rochimel Collire Avlodica } \\
\hline Model & Level (dBm) & IIS-70000 & $-113 / 6-113$ \\
\hline ILS-700** & -99 & IIS-700A & $-991-99$ \\
\hline ILS-700A & -29 & IIS-720 & $-99 /-99$ \\
\hline LS.720 & -89 & ILS-900 & $-96 /-96$ \\
\hline ILS-900 & -96 & GLU-9:2x & $-96 /-96$ \\
\hline GLU-9/x & $\$ 9$ & GNLU-9xx & $-961-96$ \\
\hline GNUU-9xx & -89 & \multicolumn{2}{|c|}{ Honeywell/Allied ShonilBendili King } \\
\hline \multicolumn{2}{|c|}{ Honeywell/Alied SignalBbenth King } & $\frac{\text { RNA-34A }}{\text { KNR-6030 }}$ & $-93 / .99$ \\
\hline RNA-34A & -87 & KNR-6030 & $-109.5 /-109.5$ \\
\hline $\mathrm{KNR}-6030$ & -93 & RIA-35A & $-103.5 /-103.5$ \\
\hline RLA-35A & -87 & RIA-35P & $-103.5 /-103.5$ \\
\hline RIA-35B & $\$ 7$ & $\mathrm{KX155, \textrm {KX156 }}$ & $-107(\max )$ \\
\hline KN 35 & $\begin{array}{l}-91.4(\mathrm{typ}) \\
87(\max ) \\
\end{array}$ & $\mathrm{KN} 35$ & \begin{tabular}{|l}
-113 (typh \\
-107 (max) \\
\end{tabular} \\
\hline \multicolumn{4}{|c|}{ VOR } \\
\hline \multicolumn{2}{|c|}{ RockweIl Colfim Avionica } & \multicolumn{2}{|c|}{$\begin{array}{l}\text { Honey well/Allied Stgmal/ } \\
\text { Beadil Klog }\end{array}$} \\
\hline Model & Level (diBm) & Model & Level(dBm) \\
\hline VOR-700** & $-107 /-107$ & RNA-34A & $-101 /-99$ \\
\hline VOR-700A & $-107 /-107$ & KNR-6030 & $-109.5 / 109.5$ \\
\hline \multirow[t]{3}{*}{ VOR-900 } & $-29 /-99$ & RVA-36A & -99199 \\
\hline & & RVA-36B & $-107 / 107$ \\
\hline & & KN 35 & $\begin{array}{l}-113 \text { (tyn) } \\
-107 \text { (max) }\end{array}$ \\
\hline
\end{tabular}

\section{Receiver Susceptibility Threshold Determination}

Several information sources on receiver interference thresholds were considered in this study. These information sources included receiver MOPS, ICAO, DO-199 and DO-233. Relevant data from these documents are extracted and summarized in this section. In addition, deficiencies in these documents are discussed and suggestions are made concerning future investigation on this topic.

Types Of Interference Considered In MOPS

Receiver MOPS RTCA/DO-192, 195 and 196 provided specifications on tolerance to various types of front end interference, or the interference caused by signals entering through the receiving antenna port. These interferences include, but are not limited to, in-band adjacent channel signal, cross modulation, intermodulation with FM broadcasts, desensitization due to high input power and out-of-band out-of-channel spurious interference. Due to low power output from PEDs (even intentional transmitters) and the high path loss between the passenger cabin and external aircraft antennas in aircraft frequency bands, those interference threats are either not relevant, or become insignificant.

The receiver MOPS, however, failed to address the most severe type of interference: the InBand On-Channel type. This type of interference is important as receivers are designed to deal with very low desired signal levels (approximately -90 $\mathrm{dBm}$ range or lower). The extreme receiver sensitivity now makes those weak in-band emissions from PEDs a concern that had to be properly addressed.

In-Band On-Channel interference are touched on only briefly in ICAO, and more extensively in DO-199 and DO-233. DO-199 and DO-233, however, are not considered as performance standards for receivers.

\section{ICAO Specifications}

The ICAO documents provided a few guidelines for receivers regarding in-band onchannel interference signal. The ICAO documents call for desired signal to undesired co-channel signal ratio to be at least $20 \mathrm{~dB}$ (ICAO Annex 10, Attachment C. Section 2.6.2.1 for Localizer, 2.5.2.2 for Glideslope and Section 3.4.6.2 for VOR). The interference signals in this case are of the same type as the desired signals, i.e. Localizer, Glideslope and VOR. However, the ICAO attachment C, where these guidelines were specified, was only intended for guidance and clarification purposes. It was not intended to be a part of the official ICAO document. Therefore, it is unlikely that these specifications were taken seriously. 


\section{DO-199 Investigation}

DO-199 and DO-233 provided the most information about receiver interference thresholds with respect to PEDs. In DO-199, many tests were conducted to determine the receiver susceptibility thresholds for various systems. Localizer, Glideslope and VOR were among the systems tested. Interference to desired signal ratio could easily be determined from the test signal strength and the measured susceptibility level.

The results of the testing reported in DO-199 were provided in the form of tables and charts, from which relevant data for Localizer, Glideslope and VOR was extracted and shown in the Table 5.

In DO-199 the official desired-to-undesired signal ratios were provided as a typical value, which is valid across most of the channel bandwidth. However, when the interfering signal is such that they beat with the local carrier to produce a frequency close to the receiver's side band, susceptibility notches can occur. The desired-toundesired signal ratio can then be as high as $38 \mathrm{~dB}$ for Localizer, $35 \mathrm{~dB}$ for Glideslope and $46 \mathrm{~dB}$ for VOR. Theoretical analysis was also conducted and presented in DO-199, and the results are shown in the same table for comparison.

According to the DO-199, it is very difficult to maintain signal lock at the susceptibility notches even if intended. The official values were therefore selected by ignoring narrowband notches. DO-199 also provided probability analysis for VOR systems to support the above observations.

A major limitation with the analysis and the susceptibility thresholds reported in DO-199 was that they were based on measurements on a single system. There were actually more than one Localizer and VOR system characterized and documented in Volume II. However, the measurements conducted on other systems were not as thorough and, therefore, not used as official data. Regardless, test results from a very limited set of equipment is a concern when extrapolating or generalizing to all products.
Table 5. Receiver Susceptibility Thresholds Reported In DO-199

\begin{tabular}{|l|c|c|c|c|}
\hline & $\begin{array}{c}\text { Test } \\
\text { Signal } \\
\text { Leve } \\
\text { (dBm) }\end{array}$ & $\begin{array}{c}\text { Disruption } \\
\text { Threshold } \\
\text { (dBm) }\end{array}$ & $\begin{array}{c}\text { Official } \\
\text { Desired to } \\
\text { Undesired } \\
\text { ratio (dB) }\end{array}$ & $\begin{array}{l}\text { Unoficial } \\
\text { Desired/ } \\
\text { Undesired } \\
\text { Ratio }\end{array}$ \\
\hline $\begin{array}{l}\text { ILS- } \\
\text { Localizer }\end{array}$ & -88 & -104 & 16 & $\begin{array}{l}38 \text { (meas.) } \\
42 \text { (theo.) }\end{array}$ \\
\hline $\begin{array}{l}\text { ILS- } \\
\text { Glide- } \\
\text { slope }\end{array}$ & -78 & -93 & 15 & 35 (meas) \\
\hline VOR & -97 & -110 & 13 & $\begin{array}{c}46 \text { (meas) } \\
51 \text { (theo) }\end{array}$ \\
\hline GPS & & -130 & & \\
\hline
\end{tabular}

\section{DO-233 Investigation}

DO-233 (Section 3.4.1) discusses Antenna Coupled Interference and the susceptibility requirements. Tests conducted by the committee have identified four interference mechanisms for IIS systems (both for Localizer and Glideslope receivers). These four mechanisms are summarized below. For convenience they are numbered as shown:

1. Mechanism 1: Out-of-Band Interference Undesired signal falling outside the frequency range of ILS receiver. Out of band interference requires too high of a level of the undesired signal to be produced by non-intentionally transmitting PEDs.

This statement was made specifically for unintentionally transmitting devices, or for out of channel emissions from intentional transmitters. For in-band intentional transmission, such as cellular phone carrier frequency, FCC spectrum management policy was supposed to provide protection in this case, according to DO-233.

\section{Mechanism 2: In-Band on-Channel "CW"} Interference:

Undesired CW signal of low level falling inside the bandwidth of selected ILS channel, but outside the sidebands of the ILS signal (susceptibility notches). In this case, interference takes place when undesired signal level is increased to $6 \mathrm{~dB}$ below the desired signal.

\section{Mechanism 3: In-Band on-Channel " $A M$ " Interference:}


Undesired signal, modulated with very low frequency of any shape, falling inside the bandwidth of selected ILS channel, but outside the sidebands of the ILS signal (susceptibility notches). In this case, the susceptibility is roughly equal to the sensitivity of the receiver, and is independent from the desired signal level. The consequence of the disturbance is unstable deviation of the ILS indicator that can lead to autopilot disconnect.

\section{Mechanism 4: Undesired signals inside the susceptibility notches:}

Undesired CW or AM signal of very low level falling inside the sidebands of the IIS signal (susceptibility notches). This phenomenon is most unlikely to occur, according to DO-233. In this case, the interference level can be as low as $40 \mathrm{~dB}$ below the desired signal level and it can result in a stable deviation of the ILS indication.

Concerning interference mechanism 1 for intentionally transmitting PEDs, protection against desensitization also depends on aircraft pathloss and PEDs carrier signal strength. According to receiver MOPS, out-of-band interference levels (desensitization) are $-13 \mathrm{dBm}$ for ILS Localizer and VOR, and $-16 \mathrm{dBm}$ for ILS Glideslope (spurious response). Thus, for cellular phones transmitting 1 watt of power $(30 \mathrm{dBm})$, a minimum pathloss of approximately $45 \mathrm{~dB}$ or lower (measured at cellular phone carrier frequency), may run the risk of interference. Realistically, cellular phones typically radiate at a much lower level than 1watt in order to conserve power and increase battery life. In addition, aircraft antennas are not designed to be efficient out of band. It is therefore expected that pathloss is significantly higher than $45 \mathrm{~dB}$ at cellular phone carrier frequencies, and the risk of interference through the antennas is low.

For ILS Localizer receiver, DO-233 sets four different interference thresholds for in-band interference. The first three deal with interference from unmodulated carrier signals, and the fourth deals with interference from a modulated carrier signal. A brief summary of the four types is shown below:

- Type I: Unwanted CW signal beats with the Localizer carrier to produce a frequency within about $0.5 \mathrm{~Hz}$ of $90 \mathrm{~Hz}$ or $150 \mathrm{~Hz}$ ILS sidebands. The unwanted RF signal must be as low as $\underline{46 \mathrm{~dB}}$ below the Localizer carrier level.

- Type II: Unwanted CW signal beats with the Localizer carrier to produce a frequency within about $10 \mathrm{~Hz}$ of $90 \mathrm{~Hz}$ or $150 \mathrm{~Hz}$ ILS sidebands. The unwanted RF signal must be as low as $26 \mathrm{~dB}$ below the Localizer carrier level.

- Type III: Unwanted CW within the ILS Localizer receiver pass band. Unwanted signal must be as low as $\underline{7 \mathrm{~dB}}$ below the Localizer carrier level

- Type IV: Unwanted "AM" modulated with $90 \mathrm{~Hz}$ or $150 \mathrm{~Hz}$. Unwanted signal must be as low as $13 \mathrm{~dB}$ below the Localizer carrier level.

While the above statements were stated explicitly for the ILS Localizer, similar statements can be made for Glideslope as well due to similarity between the two systems. DO-233 did not provide data or analysis pertaining to VOR systems' receiver susceptibility thresholds.

DO-233, however, did not provide much data to substantiate the above statements. In addition, while there were tests conducted, the number of systems tested appeared to be limited.

DO-233 is also not consistent even within itself. The statements made concerning the four interference mechanisms and the four types of interference thresholds are somewhat inconsistent with each other. An example is Type IV interference threshold versus mechanism 3 threshold for "AM" modulated interference. Type IV threshold can be as high as $13 \mathrm{~dB}$ below the Localizer carrier, while mechanism 3 threshold should be independent from the desired signal level. Also, for undesired signals inside the susceptibility notches, Type I states that the interference-to-signal ratio is as low as $-46 \mathrm{~dB}$ at the susceptibility notches, while interference mechanism 4 shows the same ratio as $-40 \mathrm{~dB}$.

There appears to be inconsistencies between DO-199 and DO-233. Measured data in DO-199 show signal to interference $(\mathrm{CW})$ of approximately $16 \mathrm{~dB}$ across the channel except near the susceptibility notches. DO-233 shows signal-tointerference of $6 \mathrm{~dB}$ to $7 \mathrm{~dB}$ for in-band on-channel " $\mathrm{CW}$ " interference. 
It is, therefore, desirable that further testing and more rigorous analysis be conducted to provide a more substantiated set of conclusions. This requires the participation of equipment manufacturers as they have the proper experience and interface equipment to deal with the issue properly.

\section{GPS Receiver Interference Threshold}

Of the four systems considered - Localizer, Glideslope, VOR and GPS - the susceptibility thresholds for GPS systems are the most well defined and consistent between various standards and regulations. A representative set of data, taken from ITU-R M.1477 [9], is summarized below.

There are three types of GPS air navigation systems in which receivers are relatively well developed:

1. Satellite Based Augmentation System (SBAS): This system is designed for Category I precision approach. Wide Area Augmentation System (GPS/WAAS) and European Geostationary Navigation Overlay Service (EGNOS) are examples of this system.

2. Ground Based Augmentation System (GBAS): This system uses ground-based pseudolite emitting signal having similar characteristics of GPS. An example is GPS/LAAS, designed for Category II/III precision approach.

3. Semi-Codeless Receiver: Receivers of this type are typically ground based and are more sensitive to interference.

Receiver susceptibility thresholds defined in ITU-R M.1477 are summarized in Table 6. Data for semi-codeless SBAS ground receivers are also provided for comparison. Even though semicodeless receivers have lower susceptibility threshold (in track mode), this type of receiver is to be used only on the ground and therefore not considered in this analysis.

ITU-R M.1477 also provided additional data concerning the behavior of the susceptibility threshold as a function of interference signal bandwidth. The narrow-band acquisition mode data shows that $\mathrm{CW}$, and for signal with bandwidth up to $700 \mathrm{~Hz}$, as the most severe threat with the lowest thresholds of $-126.5 \mathrm{dBm}$. The same threshold holds for both SBAS and GBAS air navigation receivers.

The susceptibility threshold is monotonically higher with larger interference bandwidth.

Interference threshold versus bandwidth for SBAS and GBAS air navigation receivers in track mode is similar to the data presented in Table 7. The same trend is also used for receivers in acquisition mode, with the interference threshold $6 \mathrm{~dB}$ lower. In the Table 7, narrow-band signal is defined as having bandwidth less than or equal to $700 \mathrm{~Hz}$, and wideband signal as having interference bandwidth in the range $100 \mathrm{kHz}$ to $1 \mathrm{MHz}$.

Table 6. GPS Susceptibility Thresholds

\begin{tabular}{|c|c|c|c|}
\hline & $\begin{array}{c}\text { SBAS } \\
\text { Receiver }\end{array}$ & $\begin{array}{c}\text { GBAS } \\
\text { Receiver }\end{array}$ & $\begin{array}{c}\text { Semi- } \\
\text { codeless } \\
\text { Receiver }\end{array}$ \\
\hline $\begin{array}{c}\text { Narrow-band } \\
\text { Track mode }\end{array}$ & $-120.5 \mathrm{dBm}$ & $-120.5 \mathrm{dBm}$ & $-124.5 \mathrm{dBm}$ \\
\hline $\begin{array}{c}\text { Narrow-band } \\
\text { Acquisition } \\
\text { mode }\end{array}$ & $-126.5 \mathrm{dBm}$ & $-126.5 \mathrm{dBm}$ & $-126.5 \mathrm{dBm}$ \\
\hline $\begin{array}{c}\text { Wide-band } \\
\text { Track mode }\end{array}$ & -110.5 & -110.5 & -116.5 \\
\hline $\begin{array}{c}\text { Wide-band } \\
\text { Acquisition } \\
\text { mode }\end{array}$ & -116.5 & -116.5 & -116.5 \\
$\mathrm{dBm} / \mathrm{MHz}$ & $\mathrm{dBm} / \mathrm{MHz}$ & $\mathrm{dBm} / \mathrm{MHz}$ \\
\hline
\end{tabular}

Table 7. Interference Threshold Versus Interference Bandwidth (BW) For GPS Receivers And For SBAS And GBAS Air Navigation Receivers In Track Mode.

\begin{tabular}{|l|l|}
\hline \multicolumn{1}{|c|}{$\begin{array}{c}\text { Bandwidth } \\
(\mathrm{MHz})\end{array}$} & $\begin{array}{l}\text { Receiver Interference } \\
\text { Threshold }\end{array}$ \\
\hline $0 \leq \mathrm{BW}_{\mathrm{i}} \leq 700 \mathrm{~Hz}$ & $-120.5 \mathrm{dBm}$ \\
& $\begin{array}{l}\text { Linearly increasing from } \\
-120 \mathrm{dBm} \text { to }-113.5 \mathrm{dBm}\end{array}$ \\
\hline $700 \leq \mathrm{BW}_{\mathrm{i}} \leq 10 \mathrm{kHz}$ & $\begin{array}{l}\text { Linearly increasing from } \\
-113.5 \mathrm{dBm} \text { to }-110.5 \mathrm{dBm}\end{array}$ \\
\hline $10 \mathrm{kHz} \leq \mathrm{BW}_{\mathrm{i}} \leq 100 \mathrm{kHz}$ & $-110.5 \mathrm{dBm}$ \\
\hline $100 \mathrm{kHz} \leq \mathrm{BW}_{\mathrm{i}} \leq 1 \mathrm{MHz}$ & $\begin{array}{l}\text { Linearly increasing from } \\
-110.5 \mathrm{dBm} \text { to }-97.5 \mathrm{dBm}\end{array}$ \\
\hline $1 \mathrm{MHz} \leq \mathrm{BW}_{\mathrm{i}} \leq 20 \mathrm{MHz}$ & $\begin{array}{l}\text { Linearly increasing from } \\
-97.5 \mathrm{dBm} \text { to }-91.1 \mathrm{dBm}\end{array}$ \\
\hline $20 \mathrm{MHz} \leq \mathrm{BW}_{\mathrm{i}} \leq 30 \mathrm{MHz}$ & $\begin{array}{l}\text { Linearly increasing from } \\
-91.1 \mathrm{dBm} \text { to }-89.5 \mathrm{dBm}\end{array}$ \\
\hline $30 \mathrm{MHz} \leq \mathrm{BW}_{\mathrm{i}} \leq 40 \mathrm{MHz}$ & $-89.5 \mathrm{dBm}$ \\
\hline $40 \mathrm{MHz} \leq \mathrm{BW}_{\mathrm{i}}$ & \\
\hline
\end{tabular}


In addition to the previously referenced ITU document, the following MOPS and TSO references also provide similar data for various GPS receiver systems

- RTCA/DO-208 [10] and TSO-C129a: Airborne Supplemental Navigation Equipment using GPS

- RTCA/DO-229B [11] and TSO-C146: Stand-Alone Airborne Navigation Equipment using the GPS Augmented by the Wide Area Augmentation System (GPS/WAAS)

- RTCA/DO-229A: Airborne Navigation Sensors using the GPS augmented by the Wide Area Augmentation System (GPS/WAAS)

- RTCA/DO-253A [12]: GPS/LAAS Airborne Equipment

- RTCA/DO-228 [13]: Global Navigation Satellite System (GNSS) Airborne Antenna Equipment

- RTCA/DO-235 [14]: Frequency Interference Relevant to the Global Navigation Satellite System (GNSS)

\section{General Conclusions on GPS Receiver Susceptibility}

The lowest interference threshold is -126.5 $\mathrm{dBm}$ for $\mathrm{CW}$ interfering signal and for signals having bandwidth up to $700 \mathrm{~Hz}$. The wideband interference threshold is higher. This data is consistent between many RTCA MOPS and with the ITU-R M.1477.

DO-199 also provided GPS receiver susceptibility at $-130 \mathrm{dBm}$ at the receiver. The difference of $3.5 \mathrm{~dB}$ can be easily accounted for in term of cable loss, as $-126.5 \mathrm{dBm}$ specified in receiver MOPS was provided at the output of the antenna.

It is also important to note that the susceptibility thresholds specified were given at the output of a passive GPS antenna. Thus, additional cable losses had to be considered to determine the threshold at the receiver input. If the GPS antenna is active, the GPS threshold is given at the output of the antenna, but before the pre-amplifier. Thus, the receiver susceptibility threshold and the pathloss measurement should account for the pre-amplifier gain appropriately.

\section{Estimation of Reasonable and Worst Case Minimum Receiver Susceptibility Thresholds}

It is easy to select the worst case, or "absolute minimum" receiver susceptibility threshold for the PED threat risk assessments. However, the probability analysis shown in DO-199 for VOR receiver (section 5.2.2.2) is very convincing in showing that the chance of a computer clock, or any signal, being exactly equal to $30 \mathrm{~Hz}$ VOR offset for a period long enough to cause undetectable interference is extremely unlikely (the susceptibility notches for VOR, at which the worst case interference occurs, is where the interference signal being exactly $30 \mathrm{~Hz}$ from the VOR signal). Thus, the official interference threshold for VOR used in DO-199 is $13 \mathrm{~dB}$ below the desired signal rather than the $46 \mathrm{~dB}$ worst case. This $13 \mathrm{~dB}$ signal-tointerference ratio is valid across the band except at the susceptibility notches. This value is therefore termed as a "reasonable" estimate of the ratio.

Both the DO-199 and DO-233 failed to provide a similar probability analysis for Localizer and Glideslope systems. However, the same arguments are still valid in that the probability of having any signal locks on to the susceptibility notches frequencies $(90 \mathrm{~Hz}$ or $150 \mathrm{~Hz}$ ILS sidebands) long enough to cause undetectable interference is very small. According to DO-233, the next worst case (Type II) for Localizer is when the signal beats with the Localizer carrier signal to produce a frequency within about $10 \mathrm{~Hz}$ of the 90 $\mathrm{Hz}$ or $150 \mathrm{~Hz}$ sidebands. In this case, DO-233 states that the unwanted RF signal must be as low as 26 $\mathrm{dB}$ below the Localizer carrier level. This statement can be generalized to include Glideslope systems as well due to their similarity with Localizer system. This $26 \mathrm{~dB}$ signal-to-interference ratio is therefore considered a "reasonable" estimate for both Localizer and Glideslope systems. The "worst case", defined as Type I in DO-233, has a signal-tointerference ratio of $46 \mathrm{~dB}$ for Localizer systems, and generalized to include Glideslope systems due to similarities.

Table 8 summarizes the "reasonable minimum" and the "absolute minimum" receiver susceptibility thresholds to be used for the wireless handset threat assessment reported in [3]. In this 
table, the "reasonable minimum" desired signal strength is chosen to be the minimum required sensitivity as specified in receiver MOPS such as RTCA DO-192, DO-195, and DO-196. This is the minimum receiver signal strength within the airspace coverage area, and is shown in Table 2 to be $-93 \mathrm{dBm},-86 \mathrm{~dB}$ and $-76 \mathrm{dBm}$ for VOR, Localizer and Glideslope, respectively. The "absolute minimum" desired signal strength is taken to be the lowest receiver sensitivity based on a survey of known commercial receivers shown in Table 4.

Table 8. Navigation Radio "Reasonable Minimum" and "Absolute Minimum" Interference Threshold

\begin{tabular}{|c|c|c|c|}
\hline & VOR & Loc & GS \\
\hline $\begin{array}{c}\text { Desired Signal Strength (dBm) } \\
\text { ("Reasonable min."/ "Abs. min.") }\end{array}$ & $\begin{array}{c}-93 / \\
-113\end{array}$ & $\begin{array}{c}-86 / \\
-113\end{array}$ & $\begin{array}{r}-76 / \\
-99\end{array}$ \\
\hline $\begin{array}{c}\text { - Signal/Interference ratio (dB) } \\
\text { ("Reasonable "/ "Worst case") }\end{array}$ & $\begin{array}{c}13 / \\
46\end{array}$ & $\begin{array}{c}26 / \\
46\end{array}$ & $\begin{array}{c}26 \\
146\end{array}$ \\
\hline $\begin{array}{c}\text { = Nav. Radio Min. Interference } \\
\text { Ratio (dBm) }\end{array}$ & $-106 /$ & $-112 /$ & $-102 /$ \\
("Reasonable min."/ "Abs. min.") & -159 & -159 & -145 \\
\hline
\end{tabular}

Data for GPS was consistent between various RTCA and ITU documents. The lowest interference threshold is $-126.5 \mathrm{dBm}$.

\section{Intermodulations from Multiple Wireless Devices Interaction and Threats to Aircraft Receivers}

Efforts described in [3] involve measurements of RF emission from cellular phones in several aircraft communication and navigation bands. In this effort, measurements were conducted in anechoic and reverberation chambers for RF emissions from eight CDMA, GSM and AMPS phones in four aircraft bands, including Localizer, Glideslope, VOR, and GPS. During this effort, evidence of intermodulation effects due to multiple cellular phones in the proximity of each other was unexpectedly observed.
In one set of measurements, many GSM and CDMA and AMPS phones were placed in the proximity of one another inside the test chambers (reverberation and anechoic chambers) while set at maximum transmit power. A third order intermodulation product was unexpectedly observed in the DME band, with the unwanted frequency component being as high as approximately -15 $\mathrm{dBm}$. This level was much higher than typical spurious signals emitted from the phones, which were typically in range of $-80 \mathrm{dBm}$ in the communication and navigation bands. This signal would exist only with two or more phones transmitting at the same time. Further investigation through measurement and analysis validated the intermodulation phenomenon. In addition, intermodulation products were also observed in the GPS band, but at a much lower power level. Figures 1 and 2 demonstrate the intermodulation phenomenon observed.

\section{Types of Intermodulation Interferences}

Intermodulation products are unwanted frequency components resulting from the interaction of two or more spectral components passing through a device with non-linear behavior such as a mixer, an amplifier, and output stage of a transmitter, or input stage of a receiver. The unwanted components are related to the fundamental components by sums and differences of the fundamentals and various harmonics.

For two signals, example of the intermodulation products include: $f_{1} \pm \mathbf{f}_{2} ; \mathbf{2 f} \mathbf{f} \pm \mathbf{f}_{2}$; $2 f_{2} \pm f_{1} ; 3 f_{1} \pm 2 f_{2} ;$ etc.

Intermodulation products generally fall into one of the following categories:

\section{- Transmitter Generated Intermodulation}

In this case, the transmitted signal from one transmitter is received at the output of another transmitter typically via the antenna. If the signal is of adequate strength, it will mix with the second transmitter's carrier in the non-linear final amplifier. The newly mixed signal is then amplified and transmitted along with the desired carrier. 


\section{- Receiver Generated Intermodulation}

In this case, external strong signals (two or more) algebraically mix to produce the victim frequency, usually in the first mixer or the first amplifier of the receiver. The receiver perceived the mix frequency as if it were a real signal.

\section{- Externally Generated Intermodulation}

This interference phenomenon is attributable to many sources such as dissimilar metals, dirty interconnects, loose mechanical connects, or corroded metal connections. These sources form non-linear electrical junctions, which act as "diodes" or mixers ("Rusty-Bolt" effect). When these devices are excited by one or more signals with sufficient strength, they generate intermodulation products. Electromechanical switches, tower sections with heavily corroded joints, broken welding beads are example sources of this intermodulation category.

Receiver generated intermodulation is a concern for aircraft receivers, whose antenna ports are regularly subjected to high power FM broadcasts. Many ICAO and RTCA documents were developed specifically to address this issue. Receiver MOPS, including the DO-192, 195, 196, addressed this specific type of intermodulation interference in their specifications.

In the case of intermodulation generated by multiple phones within the test chamber, it was determined to be the transmitter generated intermodulation. Combinations of filters and attenuators were used during the measurements to ensure that desensitization and intermodulation did not occur within the receiver.

\section{Demonstration of Intermodulation Products in Aircraft Bands}

As stated previously, laboratory measurements show intermodulation products from interactions of multiple phones falling within the aircraft navigation bands. Examples include a third order term $\left(2 f_{2}-f_{1}\right)$ falling inside the DME band, and a fourth order term $\left(3 f_{1}-f_{2}\right)$ falling inside the GPS band. The involved equipment includes a GSM phone and an AMPS phone, both were commanded to transmit at their maximum power. The GSM phone transmits in the frequency band allocated for use in Europe, and the AMPS phone is an example of phones using frequency band allocated for use in the U.S.

Each of the phones was commanded to transmit at a specific frequency channel, and their outputs were measured using a spectrum analyzer. A system of filters and attenuators was used to prevent undesired effects in the receiver stage. The GSM phone was transmitting at $901.9 \mathrm{MHz}$ and the AMPS (analog) phone was transmitting at $824 \mathrm{MHz}$ as measured on the spectrum analyzer. It is important to note that the frequencies measured may not match exactly with the intended frequencies (corresponding to channel numbers on the cellular phone) for transmission. The main reason was due to the uncertainty in the frequency resolution associated with the set display on the spectrum analyzer. A simple calculation showed a third order intermodulation product $2 f_{1}-f_{2}$ was 979.8 MHz, well within the DME band. In addition, a fourth order intermodulation product $3 f_{2}-f_{1}$ was computed to be $1570.1 \mathrm{MHz}$, well within the GPS interference bandwidth. Figures 1 and 2 illustrate the results measured in a reverberation chamber in comparison with prediction.

It is also observed that the third order intermodulation term in Figure 1 is at approximately $-17 \mathrm{dBm}$, which is well above spurious emission levels from any of the phones measured in aircraft band as reported in [3]. A fourth order intermodulation product in the GPS band is observed to be significantly lower, in the $82 \mathrm{~dB}$ range as shown in Figure 2.

It is generally observed that many commercial software and analysis dealing with intermodulation are more concerned with odd order intermodulation than even order ones. One reason is that odd order intermodulation products tend to be closer to one of the signals and therefore are more difficult to filter out. Figures 1 and 2 illustrates that the $3^{\text {rd }}$ order product is about $65 \mathrm{~dB}$ higher than the $4^{\text {th }}$ order product in this case. The results demonstrate that the even order products are real and should not be ignored without considering aircraft pathloss and receiver susceptibility thresholds. 